\title{
Comparing the EthoVision 2.3 system and a new computerized multitracking prototype system to measure the swimming behavior in fry fish
}

\author{
JOHANN DELCOURT, CHRISTOPHE BECCO, MARC Y. YLIEFF, \\ HERVÉ CAPS, NICOLAS VANDEWALLE, and PASCAL PONCIN \\ University of Liège, Liège, Belgium
}

\begin{abstract}
Coming from the framework of unmarked fry tracking, we compared the capacities, advantages, and disadvantages of two recent video tracking systems: EthoVision 2.3 and a new prototype of multitracking. The EthoVision system has proved to be impressive for tracking a fry using the detection by gray scaling. Detection by subtraction has given less accurate results. Our video multitracking system is able to detect and track more than 100 unmarked fish by gray scaling technique. It permits an analysis at the group level as well as at the individual level. The multitracking program is able to attribute a number to each fish and to follow each one for the whole duration of the track. Our system permits the analysis of the movement of each individual, even if the trajectories of two fish cross each other. This is possible thanks to the theoretical estimation of the trajectory of each fish, which can be compared with the real trajectory (analysis with feedback). However, the period of the track is limited for our system (about $1 \mathrm{~min}$ ), whereas EthoVision is able to track for numerous hours. In spite of these limitations, these two systems allow an almost continuous automatic sampling of the movement behaviors during the track.
\end{abstract}

Scientists study schooling or shoaling behaviors by utilizing three approaches: field studies, computer modeling, and the laboratory approach.

Field studies are complicated and rarely permit an analysis of individual positions. For example, a field study using sonar permits only a global approach with shoals (see, e.g., Axelsen, Anker-Nilssen, Fossum, Kvamme, \& Nøttestad, 2001; Nøttestad \& Axelsen, 1999). The analysis of filmed sequences in nature is very labor intensive and imprecise (see, e.g., Krause, 1993). Radio tracking is only possible for a much smaller number of individuals.

The second approach, consisting of the development of mathematical models by computer scientists (Reynolds, 1987), by biologists (Parrish, Viscido, \& Grünbaum, 2002; Suzuki, Tsumonu, \& Hiraishi, 2003; Viscido, Parrish, \& Grünbaum, 2004), or by physicists (Grégoire \& Chaté, 2004; Vicsek, Czirók, Ben-Jacob, Cohen, \& Shochet, 1995; Vicsek, Czirók, Farkas, \& Helbing, 1999) is artificial. It requires a comparison with empirical results to validate and determine the degree of validity for each model.

This research was supported by a PhD student FRIA grant and FNRS projects (2.4544.02 and 03-04-1.5.120-04).We thank Mr. Philippe Humpers and Mr. Pascal Dumont for technical assistance. We also thank L'Aquarium publique Dubuisson of the University of Liège (Belgium), particularly Richard Maréchal, Jacques Ninane, and Geoffrey Malpas. Address correspondence to J. Delcourt, Behavioral Biology Unit: Ethology and Animal Psychology, Department of Environmental Sciences \& Management, Faculty of Sciences, University of Liège, 22 Quai van Beneden, B-4020 Liège, Belgium (e-mail: johann.delcourt@ulg.ac.be).
Thanks to the development of digital imaging techniques, a third approach in the laboratory is able to detect and measure behavioral parameters - impossible to quantify with manual recording - with precision and over long time periods (Kato, Tamada, Shimada, \& Chujo, 1996; Martin, Prescott, \& Zhu, 1992; Mukhina, Bachurin, Lermontova, \& Zefirov, 2001; Nilsson, Rosén, \& Johansson, 1993; Olivo \& Thompson, 1988; Winberg, Nilsson, Spruijt, \& Höglund, 1993). For example, the EthoVision system, a commercial (Noldus Information Technology) video tracking device, was developed to measure three elements: behaviors that occur briefly and are then interspersed with long periods of inaction, behaviors that occur over many hours, and spatial measurements (e.g., distance moved, moving velocity, radial speed, or time spent in different zones of an arena) (Noldus, Spink, \& Tegelenbosch, 2001, 2002; Spink, Tegelenbosch, Buma, \& Noldus, 2001). This system has proved to be particularly efficient in the behavioral study of fish (Gerlai, 2003; Gerlai, Lahav, Guo, \& Rosenthal, 2000; Jadot, Donnay, Ylieff, \& Poncin, 2005; Ylieff, 2002; Ylieff, Bosch, Kestemont, Poncin, \& Thomé, 2003; Ylieff \& Poncin, 2003). However, this system cannot track several individuals simultaneously.

Multitracking systems are uncommon and of recent development. In EthoVision Multi-Pro, when the trajectories of two fish cross each other and their images become one, the program is unable to attribute the correct identity to each fish after they uncross (Buma, Moskal, \& Liang, 1998; Buma, Moskal, Thomas, \& Jongbloed, 1996). Suzuki et al. (2003) used another system, but without explicating its limits. Buma's team tracked up to 16 fish, and 
Suzuki's team tracked 25. Such capacities are insufficient to study the real size of shoals, which often contain hundreds of fish.

Thus, we have developed a new system of multitracking, thanks to collaboration with the Group for Research and Applications in Statistical Physics (GRASP) at the University of Liège (Belgium). GRASP has previously developed other multitracking systems for numerous (hundreds of) physical objects (e.g., bubbles or granular objects; Vandewalle, Trabelsi, \& Caps, 2004). In this article, we compare the advantages and disadvantages of our new system with EthoVision 2.3 using a concrete application: the tracking of juvenile Nile tilapia (Oreochromis niloticus L.), a weakly contrasted species that swims in shoals (often in schools) at this age.

\section{METHOD}

\section{EthoVision 2.3}

One fish-swimming freely in an aquatic arena-was filmed with a monochrome $2 / 3$ CCD video camera (see Figure 1 ). The signal from the camera was fed into a frame grabber located inside the computer, which digitized the image. Then, EthoVision analyzed the sequence image by image to detect the moving target. This detection was based on the characteristics of the animal on the screen. There were three detection methods: subtraction, gray scaling, and color. The subtraction method consists of the comparison between a reference image of the arena without an animal and each image from the experiment. The result of the subtraction represents the pixels of the movement (live). The gray-scaling method consists of the detection of all pixels in a range of gray characteristic of the animal image onscreen, and not of the arena. The color method consists of the detection of the pixels within a particular range of color that is not present in the environment. The animal is thus detected as a spot on the screen. EthoVision considers this spot only if it reaches a threshold in size that permits a filtration of noise. The computer stored three data in its memory for each image: the $x$ - and $y$-coordinates of this spot's center of gravity and the spot's size.

Using the detections by gray scaling and subtraction, EthoVision distinguishes between two animals in the same arena on the basis of size. However, in a 3-D environment, this is impossible: The apparent size of the two fish onscreen varies too much with body orientation. Moreover, this number of fish is too low to study a shoal. Detection by color theoretically permits the tracking of up to 16 objects; however, in reality, the possibilities are not so high. Ylieff (Noldus et al., 2001; Ylieff, Sanchez-Colero, Poncin, Voss, \& Ruwet, 2000) tracked three Chromis chromis (Mediterranean marine fish) in the same tank only by attaching a colored plastic pearl under the dorsal fin of each.

\section{Multitracking Prototype}

The experimental setup for this equipment was similar to that for EthoVision (see Figure 2). The detection was based on the mixing of the gray-scaling and subtraction methods. We worked with images converted to JPEG format and eliminated the background noise with a reference image of the arena without fish. Then, we detected a range of grayscale that was characteristic of the image of the fish. The detected pixels were converted to an eight-bit format (black and white images) that was usable by our program.

However, in this case the technique used to identify the numerous moving targets was different. In EthoVision, the track (displacement during a time period) is realized by the connection (over time) of the unique center of gravity of each image. In this multitracking system, this is not possible because of the large number of coordinates for each image. To obtain the track of each fish, we instead had to keep account of each movement of the fish. At the instant $t_{0}$, the fish was at the position $X_{0}$; at instant $t_{1}$, the fish was at position $X_{1}$. At instant $t_{2}$, our system detected numerous targets: Each one was potentially the tracked fish. Then the system estimated a theoretical position at the instant $t_{2}$, because we knew the direction of movement and the speed of the fish between $t_{0}$ and $t_{1}$. By this extrapolation, the computer found a theoretical point that could be compared with the real detected positions. The nearest real position to the theoretical position was attributed to the tracked fish.

This technique with feedback also permits the identification of each individual after their trajectories have crossed each other. Other parameters are automatically compared by the program - for example, the size variation of the detected targets.

\section{Concrete Example}

We tested the two video tracking systems to emphasize their advantages and disadvantages in a specific case. We attempted to track Nile tilapia juveniles (Oreochromis niloticus L.) from 2-3 weeks in age (length, $11-15 \mathrm{~mm}$ ). The fingerlings can swim freely when their

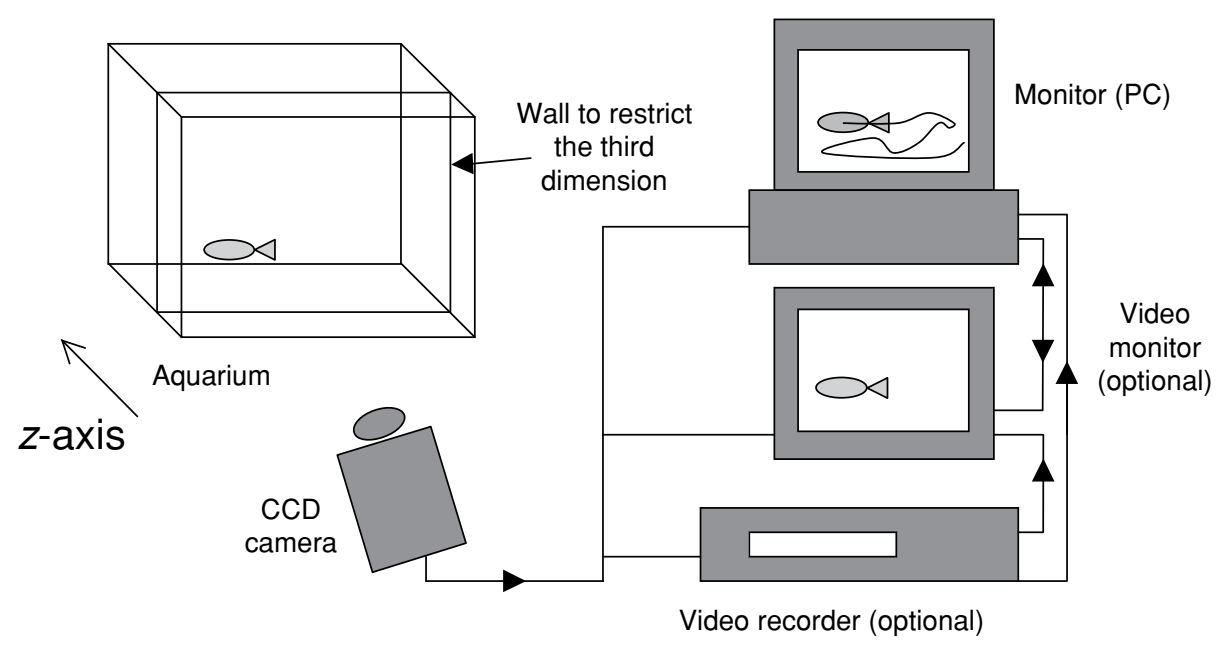

Figure 1. Diagram of the EthoVision 2.3 setup in our experiment. The analogical signal from a CCD camera is fed into a frame grabber inside the computer, which digitizes the image. EthoVision then analyzes the signal to produce quantitative descriptions of the behavior of the tracked animal. A videotape or DVD recorder and a video monitor are optional components. 


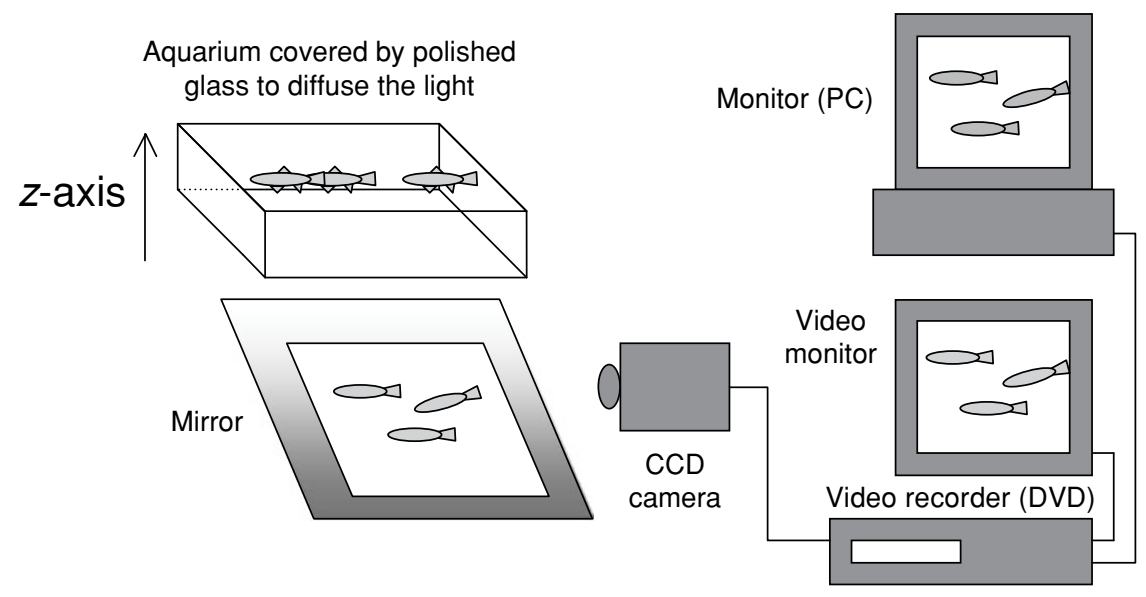

Figure 2. Diagram of the multitracking setup with a protocol similar to the EthoVision system. However, here we filmed a mirror reflecting the bottom of the tank.

yolk sac is absorbed (about 11 days after spawning), but they stay in a group near the female. These fry adopt shoaling and schooling behaviors (Mélard, 1986). These fish were reared in our laboratory (Laboratory of Fish Ethology at the University of Liège, Belgium). The breeding and experimental conditions were $12 \mathrm{~h}$ light to $12 \mathrm{~h}$ dark at $27^{\circ} \mathrm{C}$. These fry did not have an easily detectable color, and they could be marked because of their fragility.

With EthoVision, we filmed the tank with a monochrome CCD camera positioned in front of the aquarium. The dimensions of the aquarium were $($ depth) $20 \times$ (height) $20 \times$ (length) $27 \mathrm{~cm}$. These dimensions were theoretically determined according to the limit capacities of the image capture (estimated for a fish of $7 \mathrm{~mm}$, which represented 15 pixels on the PAL screen). The depth (z-axis) was reduced by a transparent Plexiglas pan that limited the displacement of the fry in this dimension $(5 \times 20 \times 27 \mathrm{~cm})$. Moreover, this pan eliminated the fish's shadows on the background. Yellow PVC pans were used in the background in order to obtain a sufficiently contrasted image of the fish. The aquatic arena was lit from above by one 58 -W fluorescent light. A blue opaque PVC pan $(27 \times 8 \mathrm{~cm})$ covered the arena so that the fish were not directly lit, increasing the contrast. We tested two detection methods: subtraction and gray scaling.

With our multitracking system, we filmed-with the same camera-a mirror placed at $45^{\circ}$ that reflected the bottom of the aquarium (dimensions $30 \times 40 \times 5 \mathrm{~cm}$ ). We limited the displacement in the third dimension with the height of water ( $z$-axis in Figure 2). The aquarium was lit from above with one 36-W fluorescent light. The aquarium was covered by polished glass to diffuse the light and to constitute the background.

\section{RESULTS}

In spite of the small size (11-15 mm) and weak contrast of the fry, we were able to follow one individual by tracking with EthoVision 2.3, as well as by subtraction and gray-scaling methods (see Figure 3). However, the apparent size of the detected target was weak in comparison with what had been theoretically estimated, knowing the capacities of the image capture card. Actually, the form (i.e., the size) of the fish and its contrast were very variable, depending on whether the fish was positioned with its face or its side to the camera. In spite of multiple adjustments, the performance proved to be different for the two methods. Though subtraction theoretically represents the best method when the background is very variable in
A

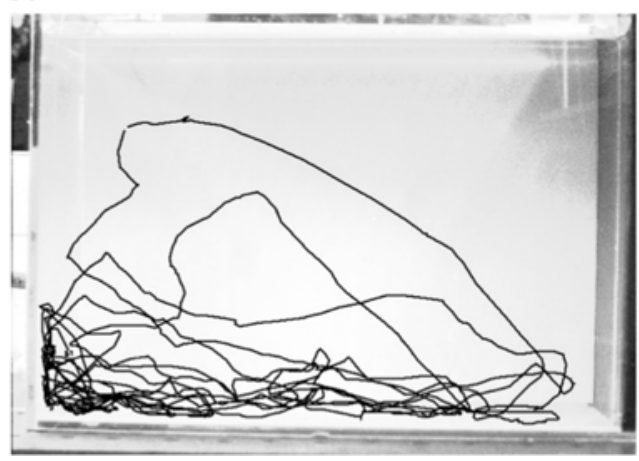

B

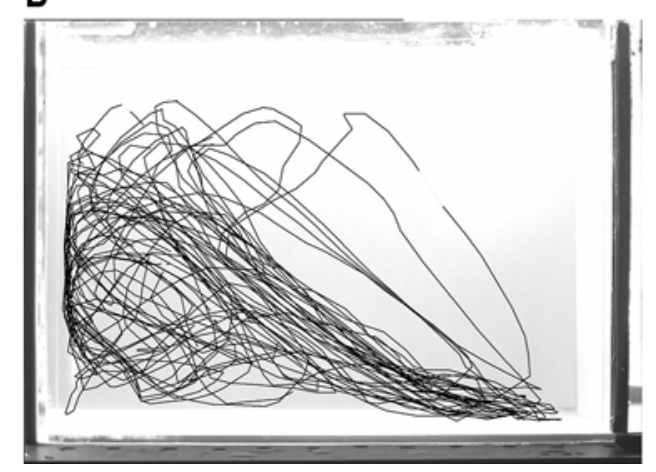

Figure 3. Examples of the EthoVision tracking of a fry for $5 \mathrm{~min}$ using the detection methods of subtraction (A) and gray scaling (B). Detection by subtraction consists of the detection of pixels with a different value of gray scaling than the self-pixels in a referent image of the arena (with no animal). Detection by gray scaling consists of the detection of pixels (target image) with a value of gray scaling significantly different from the background. 
gray level, in our application this was not the case; we obtained strong background noise. Detection was therefore limited to between 10 and 15 pixels. Consequently, the rate of loss was very important, often occurring in more than $10 \%$ of images. The loss of an image consisted of the absence of the detected target on the screen (i.e., it was under the threshold size) or a false identification (i.e., the background was the detected target).

The results for gray scaling - in spite of identical adjustments made to the camera and lighting - were better than those for subtraction. The background noise was much weaker: We were able to decrease the threshold size to the limit of the system, which is 3 pixels when the aquarium is perfectly clean. Consequently, the rate of loss of the target was much lower-less than 5\%, and often between $1 \%$ and $3 \%$.

The detection of our live target was excellent in our multitracking prototype (see Figure 4). We were able to track more than 100 fish during 2 minutes. Our program gave a number to each fish, permitting the analysis of individual behaviors if we wanted. Thus, our system allowed the study of the shoals or schools at two levels: group and individual. A feedback system that correctly identified each fish during the track, made this possible. The error probabilities were functions of the rate of images by time units. The more rapidly a camera filmed, the better the results were. However, if the fish did not move too rapidly, a rate of 25 images/sec gave very good results, without too much visual expertise from the experimenter. If the fish were too fast (i.e., during an escape response), it was better to use a high-speed camera. Moreover, when the trajectories of two fish crossed each other, the program identified the individuals after the uncrossing (see Figure 5). Although error in identification is a rare event, the human eye was necessary to detect this possibility. If such an error occurred, it was possible for the experimenter to correct it by reediting the track. The duration of the track remained short (some minutes), depending on the com- puter power needed to process the images and the numerous algorithms. The main constraint was the treatment of images before the detection of targets. In comparison, a weaker constraint was the numerous detection algorithms, which were a function of the number of targets. In fact, our system worked in two steps: First, it detected the position of each spot, then it analyzed these positions to attribute an identity to each spot.

\section{DISCUSSION AND CONCLUSION}

In spite of the difficulties of tracking fry, the two video tracking systems are complementary (see Table 1). They always present the disadvantages of any laboratory system: weaker complexity of the experimental environment, strict luminosity conditions, artificial colors of the arena (white and yellow in the present experiment), and an important confinement of the subjects. However, these systems permit the development of comparative experiments: comparisons between species; of age at different stages of development (i.e., ontogeny; Delcourt, Ylieff, \& Poncin, 2004); of the influence of various environmental characteristics (e.g., temperature [Ylieff \& Poncin, 2003] or luminosity, notably by the use of an infrared camera); or of the intrinsic characteristics of individuals (different strains [Spink et al., 2001]; parasitized individuals or xenobiotic contamination [Noldus et al., 2001; Ylieff et al., 2003]).

The two greater differences consist of the number of tracked individuals and the duration of the track. EthoVision is able to follow one unmarked fish for some hours. If the fish are marked (see, e.g., Ylieff, 2002; Ylieff et al., 2000 ), the number of tracked fish is very limited by the EthoVision Color-Pro 2.3 system. In contrast, our multitracking system permits the tracking of a higher number of unmarked fish (up to 100), more than five times the number tracked by Buma and colleagues (Buma et al., 1998; Buma et al., 1996) and Suzuki et al. (2003). However, the

A

B

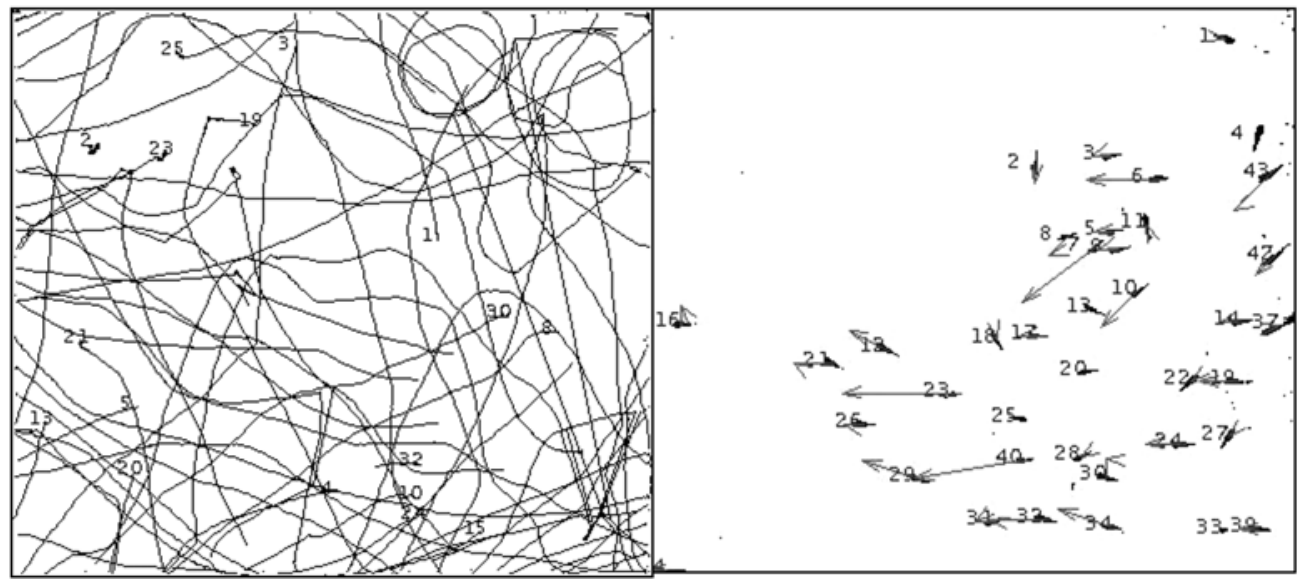

Figure 4. (A) Example of tracks with more than 30 unmarked fish. (B) Example of a picture of an analyzed sequence: Each black spot is a fish, with its speed vector. 


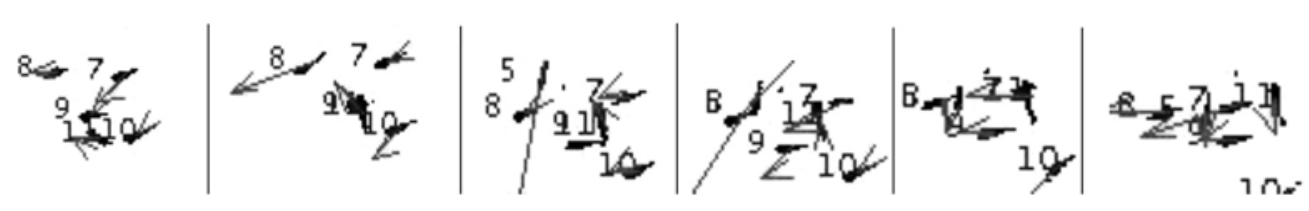

Figure 5. Sequence of multiple crossings. Fish 11 crosses Fish 9, and then Fish 7. This sequence lasts less than 0.5 sec. The program correctly and individually identifies the fish.

duration of tracking with our prototype is limited to a few minutes, due to memory capacity - mostly a consequence of the treatment of images. Consequently, if we wanted to analyze a large, filmed sequence, we would have to cut the film and assemble all data.

In our multitracking system, the group-level focus collects a greater quantity of data, permitting a better quality of statistical analysis. Actually, thanks to this system, the sampling is practically continuous, depending on the rate of the camera's image capture. Moreover, with an increase in the number of individuals, there is an increase in the amount of data. For example, the amount of data needed to statistically measure the distance of the nearest neighbor (current cohesion parameter) is equal to $N$ at each image: thus, 75,000 data items for tracking 100 fish during $30 \mathrm{sec}$ with a rate of $25 \mathrm{images} / \mathrm{sec}$. Spatial-temporal analysis is possible using the data of the position of each fish at each moment. These data can permit the determination of some structure parameters, notably cohesion and order parameters, thanks to knowledge of the positions, angles, and speed. These parameters permit direct comparisons with the computer and mathematical models elaborated by physicists (Grégoire \& Chaté, 2004; Vicsek et al., 1995; Vicsek et al., 1999) and biologists (Parrish et al., 2002; Suzuki et al., 2003; Viscido et al., 2004).
Our multitracking system can attribute a number to and monitor each fish for the whole duration of the track. This means that an analysis at the individual level is possible, in spite of having to recover the image when the trajectories of two fish cross each other, and in spite of the number of fish. The implications are interesting, notably in the chance to study the choice of particular positions by individual fish. Another application is collecting evidence for the existence of leaders in a school (see, e.g., Bumann \& Krause, 1993). However, this individual analysis is limited by the duration of the tracking. Longer filmed sequences can be analyzed by cutting, with a correct reattribution of the identification number of each fish. Of course, we are unable to identify the different individuals when we compare different filmed sequences without an indirect marking. A second limitation is error in individual identification. Although this occurrence is a rare event, human expertise is always necessary. The adjunction of a program for estimating errors of identification is possible. This system will limit the error of identification and notify the experimenter about ambiguous cases. Then, the experimenter's eye will decide the correct identification.

A great limitation of these two video tracking systems is the length of the third dimension (the $z$-axis). Our analysis was realized in two dimensions. That is why we lim-

Table 1

Comparison of the Two Video Tracking Systems in the Case of Our Experience of Fry Tracking

\begin{tabular}{l} 
EthoVision 2.3 \\
\hline Disadvantages \\
Very simplified arena and limitation of the third dimension (z-axis) of \\
our aquarium \\
Detection of only one unmarked individual by gray scaling and \\
subtraction \\
Detection by subtraction: less accurate, too much background noise (often \\
more than $10 \%$ loss of the live target, minimum target size 15 pixels) \\
Strict luminosity conditions (particularly by gray scaling)
\end{tabular}

\section{Advantages}

Detection by gray scaling: very accurate (less than $5 \%$ loss of the live target, minimum target size 3 pixels)

Long duration of tracking possible (for several hours)

Possibility of correcting the distortions caused by filming equipment

The camera allows effective filming of the front from a distance

Advantages of technique:

- very little distortion caused by filming equipment

- perspective effects are much weaker

Lighting on the top

Other Remarks

Measures the center of gravity of the image of the fish (and its size) at each frame

Multitracking

Disadvantages

Very simplified arena with limitation of the third dimension (water height, $z$-axis) and with strict luminosity conditions

Short period of tracking (some minutes)

\section{Advantages}

Detection of up to 100 unmarked fish by gray scaling

Capacity to follow and analyze the movements of

- the shoal as a whole

- each individual on the whole length of the track

Capacity to identify two individuals after their trajectories have crossed each other

The camera allows effective filming from a distance, thanks to a mirror (observation from the bottom)

Advantages of technique

- very little distortion caused by filming equipment

- perspective effects are much weaker

Diffused lighting on the top

Other Remarks

Measures of the center of gravity of each fish's image at each frame (and its size) 
ited the movement in the third dimension, to increase the precision of the measurements. However, video tracking systems that work in three dimensions have begun to appear, from the first prototype to Tracker3D (Derry \& Elliott, 1997; Laurel, Laurel, Brown, \& Gregory, 2005; Parrish et al., 2002; Pereira \& Oliveira, 1994; Viscido et al., 2004), mainly because of the coupling of some cameras. For example, Tracker3D (Viscido et al., 2004) is able to track 32 fish (with individual identification), but the duration of each track is short, and the analysis is cut into periods of $30 \mathrm{sec}$. Of course, a study in three dimensions will make it possible to obtain an incomparable degree of precision in the measuring. There will not be errors of perspective if the calibrating is well realized. Moreover, the risk of identification errors of individuals will become lower than it is in current, two-dimensional systems. And, last but not least, the environment will be more important and less confined. The animals (in this case, the fish) will be more able to adopt their normal behaviors.

Nevertheless, some of the great advantages of 2-D multitracking are the more simplified system (only one camera), and a more simplified treatment of images. In 3-D, one must perfectly synchronize the different cameras and elaborate a computer system that is able to correctly associate each spot of simultaneous images from different cameras. Two-dimensional multitracking offers the possibility to work with a circular arena and to study a bigger school. Moreover, the fry of the freshwater species often live in more shallow water in nature. Actually, the schools have properties that depend on the density of fish, particularly at high densities (Becco, Vandewalde, Delcourt, \& Poncin, 2006). At the present time-to our knowledge - only the 2-D system permits this approach. Finally, the applications are not limited to fish studies: The study of other social species is possible (e.g., a flock of birds or social insects).

\section{REFERENCES:}

Axelsen, B. E., Anker-Nilssen, T., Fossum, P., Kvamme, C., \& NøTTESTAD, L. (2001). Pretty patterns but a simple strategy: Predator-prey interactions between juvenile herring and Atlantic puffins observed with multibeam sonar. Canadian Journal of Zoology, 79, 1586-1596.

Becco, C., Vandewalle, N., Delcourt, J., \& Poncin, P. (2006). Experimental evidences of a structural and dynamical transition in fish school. Physica A, 367, 487-493.

Buma, M. O. S., Moskal, J., \& Liang, D. (1998, August). EthoVision MultiPro: Improved animal identification during automatic multiobject tracking. Measuring Behavior '98, 2nd International Conference on Methods and Techniques in Behavioral Research. Groningen, The Netherlands.

Buma, M. O. S., Moskal, J., Thomas, G., \& Jongbloed, S. (1996, October). Automatic video tracking of multiple animals without the need for marking. Paper presented at Measuring Behavior '96, 1st International Conference on Methods and Techniques in Behavioral Research. Utrecht, The Netherlands.

Bumann, D., \& Krause, J. (1993). Front individuals lead in shoals of three-spined sticklebacks (Gasterosteus aculeatus) and juvenile roach (Rutilus rutilus). Behaviour, 125, 189-198.

Delcourt, J., Ylieff, M. [Y.], \& Poncin, P. (2004, August). Measuring the ontogeny of swimming behaviour with a computerised video tracking system: Development of spontaneous activity at embryonic and early juvenile periods in the mouthbrooder Nile tilapia. Paper presented at the 2nd European Conference on Behavioural Biology. Groningen, The Netherlands.

Derry, J. F., \& Elliott, C. J. H. (1997). Automated 3-D tracking of video-captured movement using the example of an aquatic mollusk. Behavior Research Methods, Instruments, \& Computers, 29, 353-357.

GERLAI, R. (2003). Zebra fish: An uncharted behavior genetic model. Behavior Genetics, 33, 461-468.

Gerlai, R., Lahav, M., Guo, S., \& Rosenthal, A. (2000). Drinks like a fish: Zebra fish (Danio rerio) as a behavior genetic model to study alcohol effects. Pharmacology Biochemistry \& Behavior, 67, 773-782.

Grégoire, G., \& Chaté, H. (2004). Onset of collective and cohesive motion. Physical Review Letters, 92, 025702.1-025702.4.

Jadot, C., Donnay, A., YliefF, M. [Y.], \& Poncin, P. (2005). Impact implantation of a transmitter on Sarpa salpa behaviour: Study with a computerized video tracking system. Journal of Fish Biology, 67, 589-595.

Kato, S., Tamada, K., Shimada, Y., \& Chujo, T. (1996). A quantification of goldfish behavior by an image processing system. Behavioural Brain Research, 80, 51-55.

Krause, J. (1993). The relationship between foraging and shoal position in a mixed shoal of roach (Rutilus rutilus) and chub (Leuciscus cephalus): A field study. Oecologia, 93, 356-359.

Laurel, B. J., Laurel, C. J., Brown, J. A., \& Gregory, R. S. (2005). A new technique to gather 3-D spatial information using a single camera. Journal of Fish Biology, 66, 429-441.

Martin, B. R., Prescott, W. R., \& Zhu, M. (1992). Quantification of rodent catalepsy by a computer-imaging technique. Pharmacology Biochemistry \& Behavior, 43, 381-386.

Mélard, C. (1986). Les bases biologiques de l'élevage intensif du tilapia du Nil. Cahiers d'Ethologie Appliqué, 6(3).

Mukhina, T. V., Bachurin, S. O., Lermontova, N. N., \& Zefirov, N. S. (2001). Versatile computerized system for tracking and analysis of water maze tests. Behavior Research Methods, Instruments, \& Computers, 33, 371-380.

Nilsson, G. E., Rosén, P., \& Johansson, D. (1993). Anoxic depression of spontaneous locomotor activity in crucian carp quantified by a computerized imaging technique. Journal of Experimental Biology, 180, $153-162$.

Noldus, L. P. J. J., Spink, A. J., \& Tegelenbosch, R. A. J. (2001). EthoVision: A versatile video tracking system for automation of behavioral experiments. Behavior Research Methods, Instruments, \& Computers, 33, 398-414.

Noldus, L. P. J. J., Spink, A. J., \& Tegelenbosch, R. A. J. (2002). Computerized video tracking, movement analysis and behavior recognition in insects. Computers \& Electronics in Agriculture, 35, 201-227.

Nøttestad, L., \& Axelsen, B. E. (1999). Herring schooling manoeuvres in response to killer whale attacks. Canadian Journal of Zoology, 77, 1540-1546.

Olivo, R. F., \& Thompson, M. C. (1988). Monitoring animals' movements using digitized video images. Behavior Research Methods, Instruments, \& Computers, 20, 485-490.

PARrish, J. K., Viscido, S. V., \& Grünbaum, D. (2002). Self-organized fish schools: An examination of emergent properties. Biological Bulletin, 202, 296-305.

Pereira, P., \& Oliveira, R. F. (1994). A simple method using a single video camera to determine the three-dimensional position of a fish. Behavior Research Methods, Instruments, \& Computers, 26, 443-446.

REYNOLds, C. W. (1987). Flocks, herds, and schools: A distributed behavioral model. Computer Graphics Quarterly, 21, 25-34.

Spink, A. J., Tegelenbosch, R. A. J., Buma, M. O. S., \& Noldus, L. P. J. J. (2001). The EthoVision video tracking system-A tool for behavioral phenotyping of transgenic mice. Physiology \& Behavior, 73, 731-744.

Suzuki, K., Tsumonu, T., \& Hiraishi, T. (2003). Video analysis of fish schooling behavior in finite space using a mathematical model. Fisheries Research, 60, 3-10.

Vandewalle, N., Trabelsi, S., \& CAPs, H. (2004). Block-to-granularlike transition in dense bubble flows. Europhysics Letters, 65, 316-322.

VicseK, T., Czirók, A., Ben-Jacob, E., Cohen, I., \& Shochet, O. (1995). Novel type of phase transition in a system of self-driven particles. Physical Review Letters, 75, 1226-1229. 
VicseK, T., CziróK, A., FARKas, I. J., \& Helbing, D. (1999). Application of statistical mechanics to collective motion in biology. Physica A, 274, 182-189.

Viscido, S. V., Parrish, J. K., \& Grünbaum, D. (2004). Individual behavior and emergent properties of fish schools: A comparison of observation and theory. Marine Ecology Progress Series, 273, 239-249.

Winberg, S., Nilsson, G. E., SpruitT, B. M., \& Höglund, U. (1993). Spontaneous locomotor activity in Arctic charr measured by a computerized imaging technique: Role of brain serotonergic activity. Journal of Experimental Biology, 179, 213-232.

YLIEFF, M. [Y.] (2002). Validation et exploitation de nouvelles techniques d'imagerie numérique pour la caractérisation des profils comportementaux chez les poissons: Etude de l'influence de facteurs abiotiques et biotiques chez Symphodus ocellatus (Forsskål, 1775) et Chromis chromis Linné, 1758, Labridé et Pomacentridé méditerranéens. Unpublished doctoral dissertation, Université de Liège.

Ylieff, M. Y., Bosch, V., Kestemont, P., Poncin, P., \& Thomé, J. P. (2003, November). Impact of a contamination by xenobiotics (PCBS and atrazine) on the locomotor behaviour and the activity of a biomarker of exposure (EROD) in the goldfish Carassius auratus, Linnaeus, 1758. Paper presented the 10th Benelux Congress of Zoology, Leiden, The Netherlands.

YLIEFF, M. Y., \& Poncin, P. (2003). Quantifying spontaneous swimming activity in fish with a computerized color video tracking system, a laboratory device using last imaging techniques. Fish Physiology \& Biochemistry, 28, 281-282.

Ylieff, M. Y., Sanchez-Colero, C., Poncin, P., Voss, J., \& Ruwet, J. C. (2000). Measuring effects of different temperatures on swimming activity and social behavior in groups of Mediterranean marine fish with the EthoVision Color-Pro video tracking system. In Proceedings of Measuring Behavior 2000, 3rd International Conference on the Methods and Techniques in Behavioral Research (pp. 350-351). Wageningen, The Netherlands: Noldus Information Technology.

(Manuscript received May 20, 2005;

revision accepted for publication September 17, 2005.) 